\title{
Making every electron count: materials characterization by quantitative analytical scanning transmission electron microscopy
}

Matthew Weyland ${ }^{1,2}$, Zhen Chen ${ }^{3}$, Adrian J. D’Alfonso ${ }^{4}$, Yuman Zhu², Nikhil V. Medhekar², Christian Dwyer $^{5}$, Daniel J. Taplin ${ }^{3}$, Scott D. Findlay ${ }^{3}$ and Leslie J. Allen ${ }^{4}$

1. Monash Centre for Electron Microscopy, Monash University, Clayton, Victoria 3800, Australia

2. Department of Materials Science and Engineering, Monash University, Clayton, Victoria 3800, Australia

3. School of Physics and Astronomy, Monash University, Clayton, Victoria 3800, Australia

4. School of Physics, University of Melbourne, Parkville, Victoria 3010, Australia

5. Department of Physics, Arizona State University, Tempe, Arizona 85287, USA

In the past decade the ubiquity of aberration corrected transmission electron microscopes has provided a step change in the resolution and contrast attainable for the characterization of the atomic and nanoscale structure of engineering materials. Behind the scenes this instrumentation has enabled another, quieter, revolution that will be of even greater impact for the physical sciences: the move from qualitative to quantitative microscopy of atomic structure and chemistry [1]. The cornerstones to this revolution are the availability of well characterized, stable, instrumentation [2], simulation of elastic and inelastic electron scattering, numerical methods in image processing and ab initio calculation of materials energetics. These techniques are demonstrated for two different materials for both quasi-elastic electron scattering and inelastic electron scattering followed by X-ray fluorescence and collection.

The scanning transmission electron microscope (STEM) is the ideal instrument for quantitative imaging. The flexibility of the scanning probe, easy control of convergence and collection angles and the ability to collect in real and reciprocal space in the same modality enables comprehensive control of sampling in the experiment. Shown in Fig. 1 is an example application of these techniques for the solution of the structure of a new prismatic precipitate in a magnesium-calcium-indium alloy [3]. Prior studies suggested two possible structures with similar formation energies. Annular dark field (ADF) STEM images of these exceptionally thin $(5.21 \AA)$ plates reveal their distinctive structure. Image simulation of the two alternative structures, using thickness determined by averaged CBED patterns, allowed candidate 2 to be comprehensively ruled out. The resultant structure, refined by density functional theory, is fully consistent with experiments both in terms of column positions and their average intensity.

Annular dark field imaging is however not universally applicable, especially in systems with subtle differences in projected atomic number. Shown in Fig. 2 is a demonstration of a fully quantitative approach to energy dispersive X-ray (EDX) analysis across a range of thicknesses, in this case for strontium titanate $\left(\mathrm{SrTiO}_{3}\right)$ [4]. Matching simulation to experiment required inclusion of inelastic scattering via a quantum excitation of phonons (QEP) model, compensation for the efficiency of detector and determination of the exact experimental geometry of specimen, holder and detector. The precision of the thickness determination via position averaged CBED (PACBED) was improved in this study by least squares fitting to finely incremented simulation.

Quantitative imaging by STEM, across all imaging modalities and analytical signals, is now being applied to solve materials challenges across a broad range of systems. These methods promise to make optimal use of the electrons in the beam (and the X-rays generated), truly making every electron count. 
References:

[1] JM LeBeau et al, PRL 100 (2008), 206101

[2] C Dwyer et al, APL 100 (2012), 191915

[3] Y Zhu et al, Scripta Materialia 101 (2015), p. 16

[4] Z Chen et al, Ultramicroscopy 157 (2015), p. 21

[5] The authors wish to thank Ye Zhu (Monash), Nestor Zaluzec (Argonne) and Alan Sandbog (EDAX). This work was supported by the Australian Research Council (Projects DP110102228, DP140102538, DE130100739 and LE0454166) and the Monash Centre for Electron Microscopy.
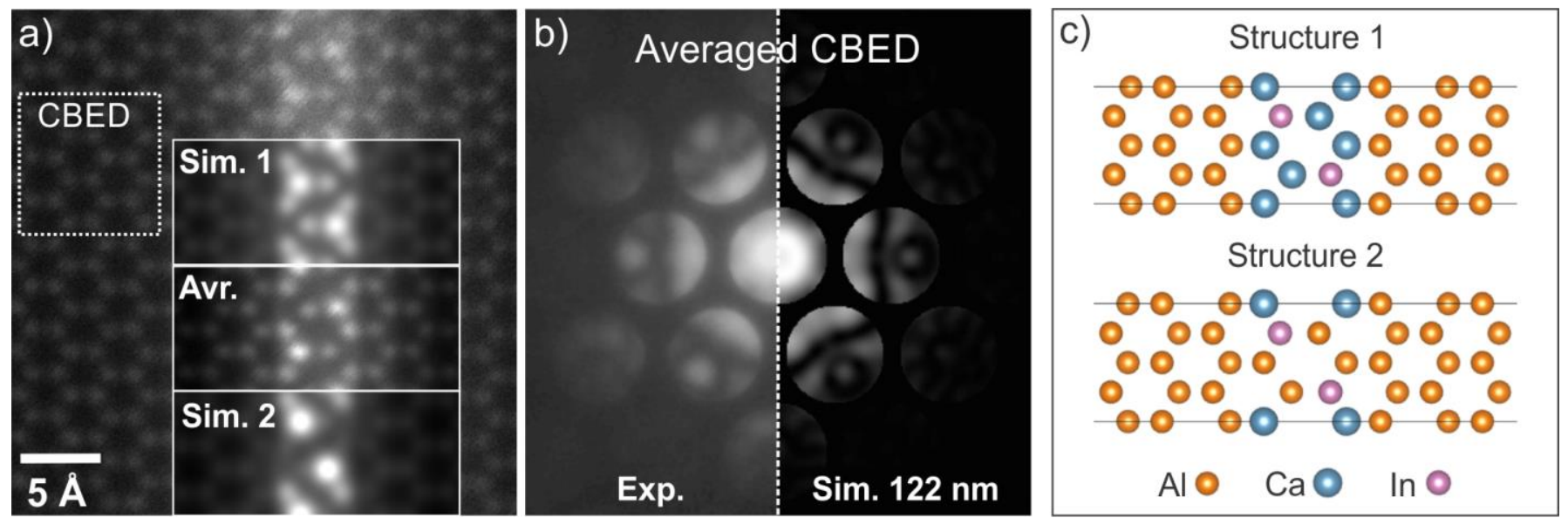

Figure 1. a) An ADF STEM image of a prismatic precipitate in $\mathrm{Mg}$, looking along the $<0001>$ direction. Indicated are simulations for two alternative structures (Sim. 1 and Sim. 2) and the lattice averaged image (Avr.). b) Averaged CBED measurement, from the dashed box in a), showing a strong match with simulation for a thickness of $122 \mathrm{~nm}$. c) The two proposed structures for the precipitate.
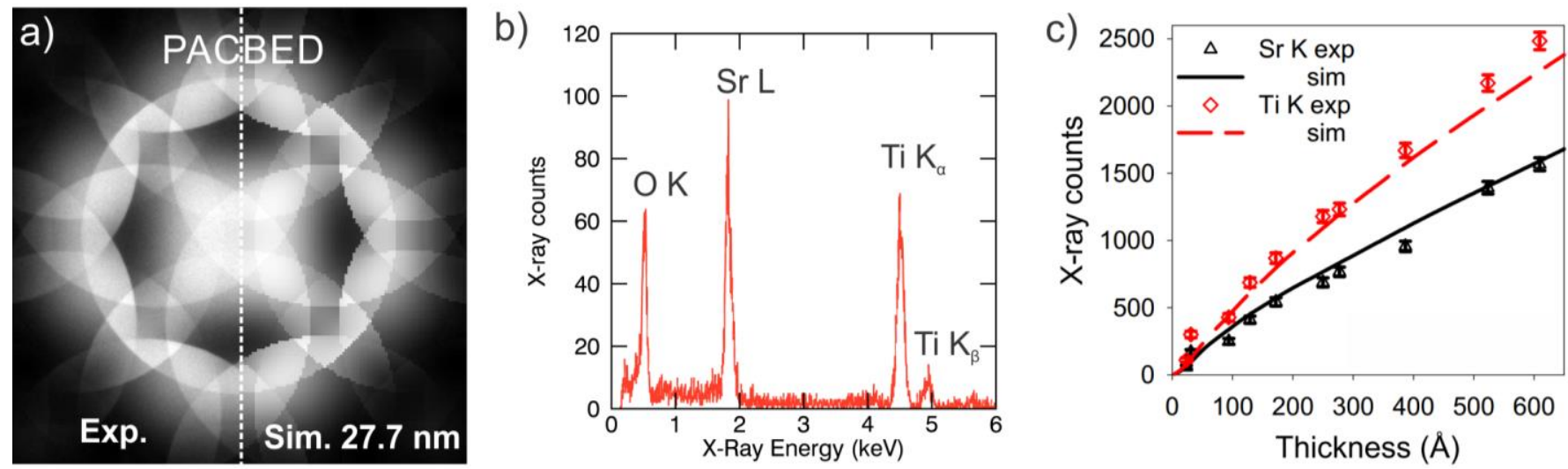

Figure 2. Quantitative EDX of $\mathrm{SrTiO}_{3}$. a) Experimental Position averaged CBED (PACBED) pattern matched with simulation. b) An EDX spectrum (reduced energy range plotted for clarity) acquired from the same area. c) A plot of PACBED thickness vs. X-ray intensity for the Sr K and Ti K peaks, showing an excellent match with simulation across a wide range of thickness. 